\title{
Extended esophagectomy in the management of carcinoma of the upper thoracic esophagus
}

Upper thoracic esophageal tumors adjacent to the trachea often require a preliminary thoracotomy to accomplish resection. Between January 1985 and July 1992, 49 consecutive patients (38 men and 11 women) underwent extended esophagectomy for esophageal cancer where the neoplasm was mobilized through an initial right thoracotomy and then resected and reconstructed through an abdominocervical approach. Ages ranged from 40 to 80 years (median 63.4 years). The tumor was located in the upper third of the thoracic esophagus in 44 patients and in the middle third in five. Thirty-three patients had squamous cell carcinoma, 14 had adenocarcinoma, and two had adenosquamous cell carcinoma. Complications occurred in 35 patients $(71.4 \%)$ and included anastomotic leak in 15, vocal cord paralysis in 11, atrial arrhythmia in nine, pneumonia in six, wound infection in five, and postoperative bleeding in one. Three patients required tracheostomy. There was one postoperative death $(2.0 \%)$. Median survival was 0.9 years (range 1 month to 5.1 years). Thirty-one patients were alive at the time this article was written, 28 without evidence of cancer. Cause of death was recurrent disease in 13 patients, unrelated to cancer in three, and unknown in one. Overall actuarial 3- and 5-year survivals were $48.6 \%$ and $18.2 \%$, respectively. Four-year survival for stage II disease was $44.6 \%$ as compared to $24.9 \%$ for stage III $(p<0.02)$. The presence of lymph node metastases significantly affected survival. Three-year survival for patients with NO disease was $77.9 \%$ compared with $20.9 \%$ for patients with N1 disease $(p<0.01)$. Age, sex, and cell type had no effect on survival. Ten patients had late dysphagia, four had gastroesophageal reflux, and one had dumping symptoms. Although associated with significant morbidity, we conclude that extended esophagectomy is an acceptable method of management for tumors of the upper thoracic esophagus. Mortality is low, and long-term results are reasonable. (J THORAC CARDIOVASC SURG 1994;107:901-7)

Wickii T. Vigneswaran, MD, Victor F. Trastek, MD, Peter C. Pairolero, MD, Claude Deschamps, MD, Richard C. Daly, MD, and Mark S. Allen, MD, Rochester, Minn.

T he goals of surgical management of carcinoma of the esophagus are cure of the disease and good long-term function and survival. These goals are best accomplished by complete resection of the cancer and reestablishment of upper digestive tract continuity. Carcinomas located in the upper thoracic esophagus adjacent to the trachea pose special problems. Frequently, the thoracic esophagus must be approached through the chest where the tumor

From the Section of General Thoracic Surgery, Mayo Clinic and Mayo Foundation, Rochester, Minn.

Read at the Seventy-third Annual Meeting of The American Association for Thoracic Surgery, Chicago, Ill., April 25-28, 1993.

Address for reprints: Victor F. Trastek, MD, 200 First St., SW, Rochester, MN 55905.

Copyright $(c) 1994$ by Mosby-Year Book, Inc.

$0022-5223 / 94 \$ 3.00+0 \quad \mathbf{1 2 / 6 / 5 2 6 2 8}$ can be safely dissected under direct vision from adjacent structures. Because a near total esophagectomy is often required, reconstruction, therefore, involves exposure of the cervical esophagus and creation of the anastomosis in the neck. Consequently, cancers located in the upper thoracic esophagus can be managed either by a right lateral thoracotomy to mobilize the esophagus and an abdominocervical approach to accomplish reconstruction, or, alternatively, by a combined abdominal, transsternal, and cervical approach. To evaluate the extended approach, we reviewed our experience with patients undergoing resection of upper thoracic esophageal carcinomas with a combined thoracic and abdominocervical approach.

\section{Patients and methods}

Between January 1985 and July 1992, 720 patients underwent esophageal resection at the Mayo Clinic for carcinoma of the esophagus. Forty-nine of these patients $(6.8 \%)$ had elective 
esophagectomy for carcinoma of the upper thoracic esophagus. The records of these 49 patients were analyzed for age, sex, symptoms, TNM classification, operative morbidity and mortality, functional results, and long-term survival. All tumors were staged after the operation by the TNM classification system of the American Joint Committee for Cancer Staging and End-Results Reporting. ${ }^{1}$ Operative deaths included patients who died within the first 30 days after operation and those who died later but during the same hospitalization. All survival probabilities were estimated by the Kaplan-Meier method with the date of esophagectomy as the starting time. ${ }^{2}$ Operative deaths and deaths that were due to all other causes were included in the survival statistics. The influence of variables on survival was analyzed by the log-rank test for discrete variables ${ }^{3}$ and the proportional hazards model of Cox for continuous variables. ${ }^{4}$ Survival for the different stages and $\mathrm{T}$ classifications was compared by a $\chi^{2}$ test with a Bonferroni correction applied to the $p$ value. A $p$ value of less than 0.05 was considered significant.

Clinical findings. There were 49 patients ( 38 men and 11 female). Median age was 63.4 years and ranged from 40 to 80 years. Signs and symptoms were present in 48 patients $(98.0 \%)$ and included dysphagia in 41 , pyrosis in 13 , chest pain in seven, anemia in six, odynophagia in five, and hoarseness in one. The carcinoma in the one patient without symptoms was discovered during surveillance for Barrett's esophagus. Twenty-two patients $(44.9 \%)$ had associated weight loss, which ranged from 2 to $23 \mathrm{~kg}$ (median, $5 \mathrm{~kg}$ ). Six patients received preoperative radiation and chemotherapy for the esophageal carcinoma.

Esophagoscopy was performed in all patients, computed tomography in 46, and bronchoscopy in 40 . Of the nine patients who did not undergo bronchoscopy, computed tomography showed no esophageal mass in six patients, and the tumor was located below the airway in three. Roentgenographic contrast examination of the colon or colonoscopy was performed in all patients for whom colon interposition was considered the most likely method of esophageal reconstruction.

The tumor was located in the upper third of the thoracic esophagus in 44 patients and in the middle third in five. Twelve patients had preoperative histologic changes of Barrett's esophagus, which extended proximal to the tumor a median of $2.8 \mathrm{~cm}$ (range 0.5 to $12.0 \mathrm{~cm}$ ). Consequently, all patients had involvement of the upper thoracic esophagus either by tumor (44 patients) or by Barrett's mucosa extending above the tumor (five patients).

All patients had preoperative mechanical bowel preparation and prophylactic antibiotics. If severe chronic obstructive pulmonary disease was present, patients were instructed to stop smoking and were trained in the use of incentive spirometry. All patients underwent initial right thoracotomy where the entire intrathoracic esophagus, cancer, and associated lymph nodes were mobilized under direct vision. After closure of the thoracotomy, the patient was placed in the supine position, and through a combined abdominal and left neck approach, an esophagectomy was performed in all patients.

Intestinal continuity was established with stomach in 46 patients and with colon in three. Reasons for colon interposition were prior total gastrectomy in two patients and prior partial gastrectomy in one. The gastric tube was passed through the esophageal bed in 36 patients and substernally in 10. A gastric emptying procedure was performed in all patients with a gastric transposition (pyloromyotomy in 43 and pyloroplasty in three).
The remaining three patients underwent reconstruction with a left colon interposition which was based on the middle colic artery in two patients and the left colic artery in one. The colon was transposed through the esophageal bed in two patients and substernally in one. When a substernal route was used to reestablish upper intestinal continuity, the head of the left clavicle and the left half of the manubrium with associated costocartilages were also excised to facilitate exposure and to avoid compression of the transplant. A feeding jejunostomy was placed in 10 patients.

An end-to-side esophagogastric anastomosis was performed with sutures in 45 patients and with staples in one. The sutured anastomoses were constructed with a single layer of interrupted sutures in 29 patients and with a double layer in 16. All three esophagocolic anastomoses were constructed with two layers of interrupted sutures. The distal end of the colon was anastomosed to the jejunum, which was prepared in a Roux-en-Y fashion in two patients and to the gastric remnant in one. Colon continuity was established with a colocolic anastomosis.

Nine patients (18.4\%) underwent additional surgical procedures that included cholecystectomy in three, pulmonary wedge excision in three, and pulmonary lobectomy for primary bronchogenic carcinoma in one, for resection of a retroperitoneal cyst in one, and for cystoscopy with urethal dilatation in one.

The cancer was classified after the operation as stage 0 in one patient, stage I in four, stage IIA in 16, stage IIB in four, and stage III in 24 . Tumor invasion was limited to the epithelium (Tis) in one patient, the submucosa (T1) in five, and the muscular wall (T2) in nine. The tumor invaded through the esophageal wall into the adventia (T3) in 26 patients and into adjacent structures (T4) in eight. Lymph node metastasis was absent (N0) in 24 and was present (N1) in 25 . Squamous cell carcinoma was present in 33 patients, adenocarcinoma in 14, and adenosquamous cell in two.

\section{Results}

There was one postoperative death (operative mortality $2.0 \%$ ) which occurred in a 54-year-old man who had bilateral pulmonary infiltrates and signs of sepsis 36 hours after operation. Because his condition deteriorated, his neck wound was reexplored. No leak or source of sepsis was identified, but the neck wound was left open. Sepsis persisted, adult respiratory distress syndrome eventually developed, and the patient died on the seventh postoperative day. Autopsy showed bilateral pneumonitis with an abscess in the left lower lobe.

Forty-two patients $(85.7 \%)$ were extubated within 24 hours. Nutritional support was instituted in 27 patients $(55.1 \%)$ and included parenteral nutrition in 16 , jejunostomy feeding in six, and both in four. The remaining 22 patients had no supplemental caloric intake.

Roentgenographic esophageal examination with the use of a water-soluble, orally administered contrast agent was performed in 45 patients, usually on the seventh postoperative day, before oral feedings; 12 had cervicomediastinal leaks. Three of the 33 patients who initially had a normal roentgenographic study had a subsequent 


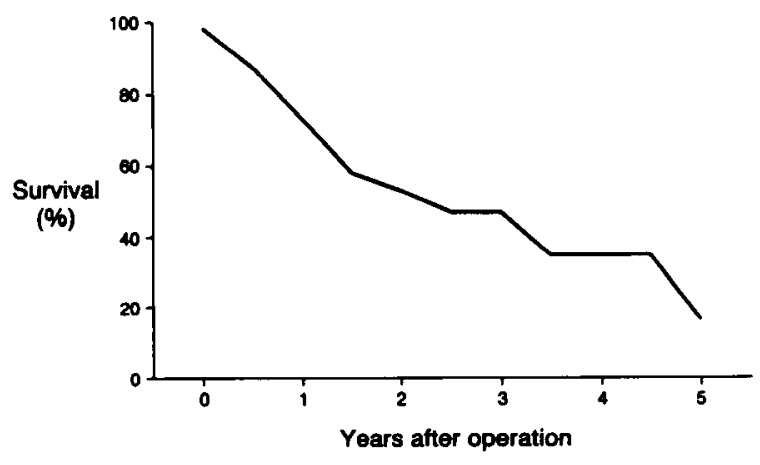

Fig. 1. Overall probability of survival (death from all causes) of 49 patients with carcinoma of the upper thoracic esophagus after extended esophagectomy. Zero time on the abscissa represents day of operation.

leak after oral feeding. Altogether, 15 patients (30.6\%) had a cervical anastomotic leak, and all occurred in patients with a gastric conduit. Leaks occurred in 5 of 10 (50.0\%) substernally placed stomachs and in 10 of 36 (27.7\%) transposed though the esophageal bed ( $p=$ not significant). Leaks also occurred in 12 of 29 single layer anastomosis ( $41.4 \%)$, in 2 of 16 double layer anastomosis $(12.5 \%)$, and in the only stapled anastomosis ( $p=$ not significant).

Thirteen of the 15 patients with cervical leaks were treated conservatively with alternative methods of nutritional support. Six of these 13 leaks were unsuspected, in that none had associated wound drainage, and the diagnosis was established by routine contrast roentgenography. All six leaks healed spontaneously; one, however, developed a late stricture. The seven other patients had evidence of leak manifested by prolonged discharge through the neck drainage catheters. Six of these leaks closed spontaneously, two of which developed late strictures. The seventh patient had dysphagia at hospital dismissal and later had a persistent enterocutaneous fistula. Median hospitalization for these 13 patients treated conservatively was 26 days (range 16 to 33 days). The remaining two patients with anastomotic leak required open drainage. One patient subsequently healed but had a late stricture; the other had a persistent enterocutaneous fistula. Hospitalization for these two patients was 24 and 25 days.

Wound infection occurred in five patients and involved the neck in four and chest in one. All four cervical wound infections were associated with anastomotic leak. Two of the four cervical wounds necessitated open drainage.

Postoperative vocal cord paralysis developed in 11 patients $(22.4 \%)$ and was unilateral in 10 and bilateral in one. Six $(54.5 \%)$ of these 11 patients had T4 tumors. One

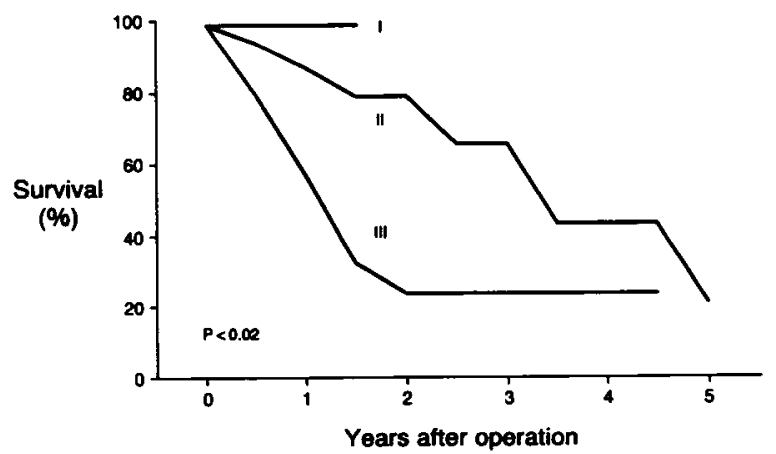

Fig. 2. Probability of survival (death from all causes) of 49 patients with carcinoma of the upper thoracic esophagus after extended esophagectomy by postoperative stage. Zero time on the abscissa represents day of operation.

patient with unilateral paralysis and the patient with bilateral paralysis had recurrent aspiration. Both required long-term jejunostomy feedings without improvement of vocal cord function. Vocal cord function returned by 6 months in eight of the remaining nine patients. The last patient required submucosal injection of Teflon into the vocal cord to restore voice.

Pulmonary complications included bacterial pneumonia in six patients with retained secretions necessitating bronchoscopy in six. Respiratory failure occurred in six patients and tracheal reintubation was required in each of them. Three of these patients eventually required tracheostomy. Two patients had chylothorax; one case resolved with conservative management, and the other required thoracotomy and ligation of the thoracic duct. One patient had postoperative bleeding from the thorax requiring reoperation. Cardiac arrhythmias occurred in nine patients. Small bowel obstruction and urinary tract infection developed in one patient each and both complications were managed conservatively.

At hospital dismissal, 45 patients were swallowing satisfactorily and were eating a soft diet. Two of the remaining patients had recurrent aspiration with swallowing. The last patient had dysphagia but did not require dilatation. One patient had gastroduodenal dumping symptoms, which resolved with dietary restrictions. Overall median hospitalization was 16 days and ranged from 9 to 115 days. Altogether, five patients required reoperation, three for anastomotic leak, one for bleeding, and one for chylothorax. Fourteen patients $(28.6 \%)$ had no complications. Median hospitalization for these 14 patients was 12.5 days and ranged from 9 to 18 days.

Survival. Follow-up was complete for 47 of the 48 operative survivors and ranged from 1 month to 5.1 years (median, 0.9 years). No follow-up was available in one 


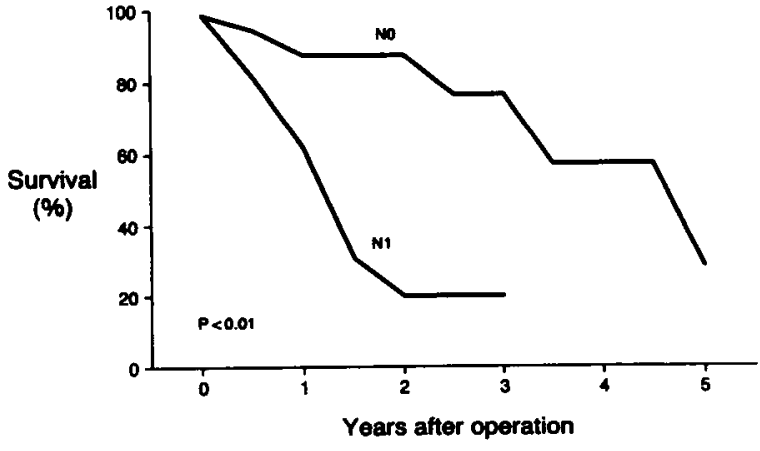

Fig. 3. Probability of survival (death from all causes) of 49 patients with carcinoma of the upper thoracic esophagus after extended esophagectomy by $\mathrm{N}$ classification. Zero time on the abscissa represents day of operation.

patient. Sixteen patients had postsurgical adjuvant therapy, 11 had radiation therapy, and five had both radiation and chemotherapy. Ten patients with $\mathrm{N} 1$ disease, five with T4 lesions, and one with N0 disease received adjuvant therapy.

Recurrent tumor was found in 16 patients (34.0\%). The site of recurrence was disseminated in seven, regional in five, anastomotic in three, and unknown in one. Further treatment for recurrent cancer was done in 10 patients and included radiation therapy in six, chemotherapy in two, both in one, and radiation and further operation in one.

Thirty-one patients were alive at last follow-up. Median survival in these 31 patients was 0.9 years and ranged from 38 days to 5.1 years. Twenty-eight of these patients had no evidence of recurrence. Median survival in the 17 patients who died was 0.8 years and ranged from 50 days to 4.6 years. Cause of death was recurrent cancer in 13 , unrelated to cancer in three, and unknown in one. None of the deaths unrelated to cancer involved known recurrence at the time of death.

Overall actuarial 3- and 5-year survivals (Fig. 1) were $48.6 \%$ and $18.2 \%$, respectively, and varied according to TNM classification (Fig. 2). Four-year survival for combined stage II disease was $44.6 \%$, compared with $24.9 \%$ for stage III $(p<0.02)$. The presence of lymph node metastases significantly affected survival. Three-year survival for patients with N0 disease was $77.9 \%$, compared with only $20.9 \%$ for patients with N1 disease $(p<0.01)$ (Fig. 3). Age, sex, and cell type had no effect on survival. Patients with $\mathrm{T} 3$ tumors (median survival 1.5 years) was less favorable than that observed in patients with either T1 or T2 tumors (median survival 4.8 years), but this difference was not significant $(p=0.06)$. The presence of a postoperative complication, however, sig- nificantly affected survival. Four-year survival for the 14 patients who had no complications was $90.9 \%$, compared with $25.0 \%$ survival observed for the 34 patients with complications $(p<0.05)$. Patients who had no complications tended to be younger ( 58.4 years versus 64.5 years for those with complications) and to have less advanced cancer ( $56 \%$ stage I and II versus $44 \%$ for those with complications). These differences, however, were not significant.

Late morbidity and functional results. Ten patients $(20.8 \%)$ had mild to severe dysphagia which was a result of benign stenosis in eight patients and recurrent carcinoma in two. Four patients required dilatations. One of the malignant strictures subsequently underwent stent placement. Six patients with mild dysphagia did not require dilatation.

Four patients had gastroduodenal dumping symptoms, and four had late reflux symptoms. All were medically treated. One patient had late hoarseness as a result of local recurrence. Other late complications, each in one patient, included anorexia, bloating, and massive hematemesis from anastomotic recurrence.

\section{Discussion}

The goals of surgical management of esophageal cancer are complete resection of the tumor, palliation of dysphagia by reestablishment of upper digestive tract continuity, and long-term survival. These goals should be accomplished with the lowest mortality and morbidity possible and should provide the best long-term function. Mr. Ivor Lewis, at the Hunterian lecture in 1946, echoed this philosophy when he stated: "The aims of radical cancer surgery are: (a) to cure the disease while (b) rendering the patient's life useful and enjoyable—or at least bearable."5

The treatment of upper thoracic esophageal cancers is challenging. The intimate relationship of the esophagus to the airway, arch of the aorta, and recurrent laryngeal nerve poses special technical problems. Extended esophagogastrectomy allows direct visualization of the mediastinal structures and permits reconstruction with a cervical anastomosis. This procedure has been promoted by Akiyama and associates ${ }^{6}$ and McKeown. ${ }^{7}$ Alternatively, Ong and associates ${ }^{8}$ and Orringer ${ }^{9}$ have described a combined abdominal, transsternal, and cervical approach with the patient in a supine position. Transhiatal esophagectomy is also possible but carries increased risk of tumor perforation and vascular and airway injury when bluntly mobilizing the esophagus from the mediastinal structures. ${ }^{10,11}$ The classic Ivor Lewis esophagogastrectomy ${ }^{5}$ allows direct visualization of the mediastinal structures but limits the proximal resection margin. 
In the current series, operative mortality was $2.0 \%$ and is similar to our experience with Ivor Lewis esophagogastrectomy ${ }^{12}$ and transhiatal esophagectomy for carcinomas of the lower esophagus. ${ }^{13}$ This mortality is less than the operative mortalities of Ong and associates 8 and McKeown ${ }^{7}-17.6 \%$ and $13.8 \%$, respectively; and it is similar to that of Akiyama and associates $6-1.4 \%$.

Significant morbidity occurred in over two thirds of our patients and included anastomotic leak, recurrent laryngeal nerve injury, and pulmonary complications. Approximately one third of our patients had anastomotic leaks. Although the majority resolved with an alternative method of nutrition and cervical drainage, $13 \%$ did require surgical intervention. Hospitalization was longer in those patients with anastomotic leaks, and $40 \%$ eventually had long-term sequelae, including fistula and stricture. A high prevalence of anastomotic leaks has been reported in patients with cervical anastomosis by us and others. ${ }^{13-16}$ We do not believe, however, that this high prevalence is the result of poor anastomotic technique. We previously reported ${ }^{12} 100$ consecutive Ivor Lewis esophagogastrectomies with only nine leaks, six of which were not clinically significant. Instead, cervical anastomotic leaks may be due to increased tension and to the additional length of stomach required for reconstruction with further potential for compromise of the conduit's blood supply. In addition, the narrow thoracic inlet through which the stomach must be transposed may also play a role by interfering with venous return, causing congestion and decreased healing. Lastly, the gastric tube can be constructed in a variety of ways. In this study, the tube was made along the greater curve, sacrificing the majority of the lesser curvature. This may have interfered with the submucosal vascular plexus along the lesser curve and led to a higher leak rate. ${ }^{17}$

Recurrent laryngeal nerve injury occurred in approximately one fifth of our patients and contributed to pulmonary complications. In many of these patients, vocal cord injury was not permanent and improved by the sixth postoperative month. More than half of our patients with this complication had tumor extension beyond the esophageal wall. Because of the proximity of the recurrent laryngeal nerve to the tumor, it was, at times, necessary to excise the nerve to obtain complete resection of the tumor. When tumor is not involving the nerve, careful dissection and the avoidance of a metal retractor on the trachea will reduce recurrent nerve injury.

In our experience, preoperative computed tomography was invaluable in localizing the tumor and in assessing its relationship to the airway, ascending aorta, and azygous vein. When no evidence of esophageal thickening was present adjacent to the airway, it was unlikely that tumor invasion of either the airway or recurrent laryngeal nerve was present. Preoperative bronchoscopy is also important to evaluate tracheal invasion. If esophageal cancer is found to be invading the trachea, curative resection is contraindicated. Esophagoscopy is helpful not only to confirm the diagnosis of cancer but also to determine the presence and length of associated Barrett's disease and evaluate the stomach. If Barrett's epithelium extends proximal to the cancer in midesophageal tumors, we routinely make the anastomosis in the neck. We believe that surgical management of these patients must include resection of all Barrett's epithelium. This was the situation in $10 \%$ of our patients, all of whom had a midthird esophageal cancer with proximal Barrett's disease.

Our 5-year survival for extended esophagectomy for carcinomas of the upper thoracic esophagus was $18.2 \%$ and nearly identical to that which we observed after Ivor Lewis $(22.8 \%)$ and transhiatal esophagogastrectomy (20.8\%) for carcinomas of the lower esophagus and gastroesophageal junction. ${ }^{12,13} \mathrm{We}$ continue to find that survival is stage dependent. Likewise, late function was good; more than $75 \%$ of our patients had no dysphagia at follow-up, which was similar to that observed for patients with lower esophageal cancers. ${ }^{12}$

In conclusion, extended esophagectomy is indicated in those patients with upper thoracic esophageal tumors immediately adjacent to major airways. In addition, this procedure is a good method of management in selected patients with midesophageal cancers who have proximal Barrett's disease. In our experience, extended esophagectomy for malignant esophageal disease was performed with low mortality and good late function. Survival was stage dependent and similar to that reported for tumors of the lower esophagus. Although morbidity was significant, we continue to use this extended approach for neoplasms of the upper thoracic esophagus.

\section{REFERENCES}

1. Beahrs OH, Myers MH. American Joint Commission on Cancer: manual for staging of cancer. 2nd edition. Philadelphia: Lippincott, 1983:178.

2. Kaplan EL, Meier P. Non-parametric estimation from incomplete observation. JASA 1958;53:457-81.

3. Peto R, Peto J. Asymptotically efficient rank and invarient procedures (with discussion). J R Stat Soc A 1972;135:185201.

4. Cox DR. Regression models and life-table (with discussion). J R Stat Soc B 1972;34:187-220.

5. Lewis I. The surgical treatment of carcinoma of the esophagus with special reference to a new operation for growth of the middle third. Br J Surg 1946;34:18-31.

6. Akiyama H, Tsurumaru M, Kawamura T, Ono Y. Princi- 
ples of surgical treatment for carcinoma of the esophagus. Ann Surg 1981;194:438-46.

7. McKeown KC. The surgical treatment of carcinoma of the oesophagus: a review of the results in 478 cases. J R Coll Surg Edinb 1985;30:1-14.

8. Ong GB, Lam KH, Lam PHM, Wong J. Resection for carcinoma of the superior mediastinal segment of the esophagus. World J Surg 1978;2:497-504.

9. Orringer MB. Partial median sternotomy: anterior approach to the upper thoracic esophagus. J THORAC CARDIOVASC SURG 1984;87:1249.

10. Fok M, Siu KF, Wong J. A comparison of transhiatal and transthoracic resection for carcinoma of the thoracic esophagus. Am J Surg 1989;158:414-9.

11. Orringer MB, Orringer JS. Esophagectomy without thoracotomy: A dangerous operation? J THORAC CARDIOvaSC SURG 1983;85:72-80.

12. King RM, Pairolero PC, Trastek VF, Payne WS, Bernatz PE. Ivor Lewis esophagogastrectomy for carcinoma of the esophagus: early and late functional results. Ann Thorac Surg 1987;44:119-22.

13. Vigneswaran WT, Trastek VF, Pairolero PC, Deschamps C, Daly RC, Allen MS. Transhiatal esophagectomy for carcinoma of the esophagus. Ann Thorac Surg 1993; $56: 838-46$

14. Shahian DM, Neptune WB, Ellis FH Jr, Watkins E Jr. Transthoracic versus extrathoracic esophagectomy: mortality, morbidity, and long-term survival. Ann Thorac Surg 1986;41:237-46.

15. Hankins JR, Attar S, Coughlin TR Jr, et al. Carcinoma of the esophagus: a comparison of the results of transhiatal versus transthoracic resection. Ann Thorac Surg 1989;47: $700-5$.

16. Chassin JL. Stapling technique for esophagogastrostomy after esophagogastric resection. Am J Surg 1978;136:399404.

17. Collard JM, Otte JB, Jamart J, Reynaert M, Kestens PJ. An original technique for lengthening the stomach as oesophageal substitute after oesophagectomy: preliminary results. Dis Esoph 1989;2:171-4.

\section{Discussion}

Dr. Stanley C. Fell (Bronx, N.Y.). The Mayo Clinic group is to be congratulated for their excellent results with the procedure described by McKeown. ' We have used this procedure as well but have generally used a modification of a technique described by Akiyama, ${ }^{2}$ which has certain virtues.

With the patient supine, a two-team approach mobilizes the stomach and the cervical esophagus. Stapling and transection are performed, and the stomach is passed retrosternally, and anastomosis performed to the cervical esophagus. Resection of the sternum or clavicle is not required. Rather, the strap muscles and the sternal head of the sternomastoid muscle are transected. The cervical and abdominal wounds are closed, the patient is placed in the left lateral decubitus position, and the entire thoracic esophagus is resected via a right thoracotomy with work being done from both ends.

A significant advantage of this technique is that the stomach is not in the tumor bed. Catheters are inserted for brachytherapy, and the patient receives external beam therapy as well. The main problem, of course, is the poor margin on the membranous trachea. We have had some encouraging results, with a few long-term survivors.

\section{REFERENCES}

1. McKeown, KC. Total three-stage esophagectomy for cancer of the esophagus. Br J Surg 1976;63:259.

2. Akiyama, H. Surgery for carcinoma of the esophagus. Current Prob Surg 1980;17:87-9.

Dr. Joseph I. Miller (Atlanta, Ga.). Before Dr. Ginsberg left Toronto, as I understand it, the group there had stopped operating on carcinoma that was above the level of the aortic arch and was using combined chemotherapy and radiation only for lesions that would be approximately 20 to $22 \mathrm{~cm}$ or at least above the level of the superior aortic arch. Do you have any thoughts about this? Also, would Dr. Orringer, from the University of Michigan, comment about his feelings about operating for primary carcinoma of the esophagus at this level.

Dr. Vigneswaran. With regard to Dr. Fell's question, we placed the esophageal conduit in the substernal position in 11 patients who were, at the time of operation, thought to have residual cancer in the esophageal bed. This procedure is, however, not our routine practice in patients who are deemed to have had complete resection of the cancer.

The choice of treatment for upper esophageal carcinoma is controversial. I agree with Dr. Miller that some centers would have the oncologist treat these patients. Our experience indicates that an extended esophagectomy can be performed for upper thoracic esophageal cancer with low operative mortality and a 5 -year survival of approximately $20 \%$. This outcome is similar to lower or midesophageal carcinoma after surgical resection. We therefore believe that extended esophagectomy is the choice of treatment at present for localized upper thoracic esophageal carcinoma.

Dr. Mark B. Orringer (Ann Arbor, Mich.). Fortunately, upper third thoracic esophageal tumors are least frequent in occurrence because their prognosis is the worst of all thoracic esophageal carcinomas. We have only one 5-year survivor of upper third esophageal cancer in our entire series of resections over the past 20 years. These tumors are horrible actors because of the typical extent of mediastinal involvement, both lymphatic and direct invasion, when they are first seen. I would like to remind the audience that the addition of a partial upper sternal split allows access to the high retrotracheal esophagus and exposure and mobilization of these tumors under direct vision after which a transhiatal esophageal resection and cervical esophagogastric anastomosis can then be completed. While there is always a concern about tracheobronchial invasion with these cancers, if there is no evidence of this at bronchoscopy, resection and reconstruction as I just described is generally possible.

Would you comment further on your experience with cervical esophagogastric anastomotic leaks. I personally have grave concern about the conservative management of these leaks, even in the patient without symptoms. We are all influenced by our worst anecdotal case, but I had the unfortunate experience of discharging a patient without symptoms with a contained cervical esophagogastric anastomotic leak only to have him return 
10 days later with erosion of his undrained cervical abscess through the membranous trachea, resulting in a gastrotracheal fistula that ultimately led to his death. It is so much easier and safer to open the neck wound at the bedside and treat these leaks with wound irrigations with swallowed water and packing that conservative management seems unnecessarily risky. I would be interested to learn approximately what percentage of your cervical esophagogastric anastomotic leaks were successfully managed conservatively and whether there were any catastrophic long-term complications like the one I mentioned.

Dr. Vigneswaran. The patients who were treated conserva- tively for anastomotic leak had no symptoms except for prolonged drainage from the cervically placed drain. These patients remained in the hospital until the drainage was minimal. None were dismissed from hospital with apparent residual infection or leak. One patient in this group, however, had a cervical esophago-cutaneous fistula, indicating that this patient may have had ongoing leak or infection not obvious at the time of dismissal. The remaining patients treated conservatively did well at follow-up except for late dysphagia in three patients. We do not believe an aggressive management of this subgroup of patients with anastomotic leak is warranted.

\section{Bound volumes available to subscribers}

Bound volumes of THE JOURNAL OF THORACIC AND CARDIOVASCULAR SURGERY are available to subscribers (only) for the 1994 issues from the Publisher, at a cost of $\$ 82.50$ for domestic, $\$ 106.28$ for Canadian, and $\$ 100.50$ for international subscribers for Vol. 107 (January-June) and Vol. 108 (July-December). Shipping charges are included. Each bound volume contains a subject and author index and all advertising is removed. Copies are shipped within 60 days after publication of the last issue of the volume. The binding is durable buckram with the JOURNAL name, volume number, and year stamped in gold on the spine. Payment must accompany all orders. Contact Mosby-Year Book, Inc., Subscription Services, 11830 Westline Industrial Drive, St. Louis, Missouri 63146-3318, USA; phone 1 (800) 4534351 or (314) 453-4351.

Subscriptions must be in force to qualify. Bound volumes are not available in place of a regular JouRNAL subscription. 